\title{
LA ESCRITURA, ¿UNA POSIBLE HERRAMIENTA EN LA PREVENCIÓN DEL SUICIDIO?
}

\author{
WRITING, A POSSIBLE TOOL FOR SUICIDE PREVENTION?
}

\author{
Miriam Castellanos-Lozano* \\ Universidad de Monterrey \\ Monterrey-México \\ Hada Soria-Escalante \\ Universidad de Monterrey \\ Monterrey-México \\ Recibido noviembre de 2019/Received November, 2019 \\ Aceptado septiembre de 2020/Accepted September, 2020
}

\begin{abstract}
RESUMEN
Alrededor de 800.000 personas cometen suicidio en el mundo año tras año (Organización Mundial de la Salud [OMS], 2019). Esto ha traído como consecuencia la necesidad de desarrollar programas preventivos; sin embargo, estos programas se centran principalmente en los métodos comunes de suicidio y la psicoeducación. De acuerdo con la Organización Mundial de la Salud (OMS, 2014), a pesar de que se han elaborado distintos programas preventivos, estos esfuerzos no se han desarrollado en todos los países debido a la falta de sensibilización acerca de la importancia de la prevención del suicidio entre sus autoridades y la sociedad en general, así como por la falta de implementación de los programas existentes. Haciendo uso de la perspectiva psicoanalítica respecto de la escritura y la creatividad, el presente trabajo tiene por objetivo explorar la posibilidad de la escritura como una herramienta distinta en el marco de la clínica de la prevención, la que tiene como base la teoría psicoanalítica predominante. Mostramos la escritura como proceso de sublimación psíquica y asunción simbólica del sujeto, que pone en evidencia el dolor psíquico de quien escribe, la escritura como una posibilidad terapéutica ante el sujeto movido por el suicidio, comandada por quien escribe, donde el propio sujeto se hace cargo de su historia y su producción singular en su totalidad. Recurrir a la escritura como una vía en donde se pueda accederse al entendimiento de los procesos psíquicos de las personas, podría apuntar hacia la identificación de personas en riesgo y a la prevención del suicidio.
\end{abstract}

Palabras Clave: Escritura, Suicidio, Prevención, Psicoanálisis, Psicopatología.

\begin{abstract}
Around 800,000 people commit suicide in the world year after year (World Health Organization [WHO], 2019). This has resulted in the need to develop preventive suicide programs; however, these programs mainly focus on the common suicide methods and psychoeducation. According to the World Health Organization (WHO, 2014), although different preventive programs have been developed, these efforts have not been developed in all countries due to the lack of awareness about the importance of suicide prevention among their authorities and society in general, as well as the lack of implementation of these programs. Through the psychoanalytic perspective about writing and creativity, this paper aims to explore the possibility of writing as a different tool in the suicide prevention, which is based on the predominant psychoanalytic theory. Writing is displayed as a psychic sublimation process and symbolic assumption of the subject which shows the psychic pain of the person who writes; writing as a therapeutic possibility for the person with suicide thoughts. The subject assumes a predominant role on his or her story and his or her singular production as a whole. Writing as a way to access to understanding of the psychic processes of people, could lead to the identification of people at risk and suicide prevention.
\end{abstract}

Key Words: Writing, Suicide, Prevention, Psychoanalysis, Psychopathology. 


\section{EL SUICIDIO: UNA PROBLEMÁTICA MUNDIAL}

El suicidio es definido como acto deliberadamente iniciado por el fallecido con la expectativa de un resultado fatal (la muerte), y mediante esto la persona pretende obtener los cambios que desea (Campo-Arias y Suárez-Colorado, 2019; Cervantes y Melo, 2008; Organización Mundial de la Salud [OMS], 2001). El comportamiento suicida abarca: la ideación suicida, la que en muchas ocasiones la comunicación de la intención es manifestada de manera verbal o no verbal; el plan de suicidio; el intento de suicidio; y la consumación del suicidio (OMS, 2001).

En la actualidad, el suicidio constituye una compleja problemática que se relaciona con múltiples causas, siendo las enfermedades mentales uno de los factores más relevantes en la predisposición de este comportamiento, porque quienes son afectados por enfermedades mentales presentan un riesgo diez veces mayor en comparación con aquellos que no las padecen (Cervantes y Melo, 2008; Córdova, Rosales y Rosales, 2018; Ministerio de Salud del Gobierno de Chile, 2013; Urrego, Quintero y Manrique, 2016). Sin embargo, otros factores tanto individuales como contextuales forman parte del desarrollo del acto suicida.

Estimar el grado de intencionalidad de quienes presentan un comportamiento suicida no es sencillo (Mann et al., 2005; OMS, 2001). En ocasiones, algunas conductas relacionadas al suicidio pueden presentarse con un objetivo distinto, lo que señala que pudieran existir muertes accidentales que puedan ser concluidas como un suicidio aun cuando su primera intención no fuera el terminar con la vida (Fernández-Cabana, 2015; Klonsky, May y Saffer, 2016; Klonsky, Saffer y Bryan, 2018). Ante esto, y de manera que se apunte hacia la prevención del suicidio, debe hacerse hincapié en la comprensión del origen de la conducta suicida para poder generar intervenciones más efectivas (Fisher, Medaglia y Jeronimus, 2018; Schwartz-Lifshitz, Zalsman, Giner y Oquendo, 2012).

Cerca de 800.000 personas se suicidan al año. Es decir, entre una y dos personas se suicidan por minuto en el mundo. El grupo con mayor incidencia pertenece al sector socioeconómico bajo y medio, lo que corresponde al $79 \%$ de los suicidios que se presentan anualmente, aunque la tasa más elevada (11,5 por 100.000 habitantes) se presenta en los países de alto grado de desarrollo. El número de suicidios año tras año se ha incrementado de tal manera, que en el 2016 el suicidio fue considerado la principal causa de muerte en jóvenes de entre 15 y 29 años (OMS, 2019). Ante lo anteriormente mencionado, es menester considerar la prevención de intentos de suicidios como un desafío para los profesionales del sector salud y la sociedad en general.

En todo el mundo se han desarrollado programas de prevención ante el incremento de suicidios en los últimos años, sin embargo, estos se centran en su mayoría en brindar información para la identificación de los indicadores clínicos del riesgo suicida, o de la evaluación de signos específicos de depresión mayor, asociados al riesgo suicida. Asimismo, los programas preventivos actuales, en su mayoría, se centran en desarrollar los factores de protección, así como redes de apoyo, desarrollo de habilidades personales, etcétera, y pocos se encargan de implementar herramientas que puedan facilitar la identificación de personas en riesgo de suicidio. Esto señala que la implementación de estrategias de prevención requiere de mayor investigación, así como el uso de estrategias integrales e integradas que realmente sean efectivas en cuanto a la prevención e identificación de conductas suicidas (Zalsman et al., 2016).

La OMS (2001) señala que la prevención del suicidio debe comprender actividades que abarquen la provisión de mejor educación, tratamiento eficaz de los trastornos mentales y el control medioambiental de los factores de riesgo, así como la difusión apropiada de información y la sensibilización de la sociedad en general. Aunado a lo anterior, la estigmatización de los trastornos mentales, y particularmente del suicidio, resulta ser un elemento que disuade a las personas de buscar ayuda para aquellos que piensan en el suicidio o que han tenido intentos de ello, limitando su acceso a las intervenciones pertinentes (Campo-Arias y Suárez-Colorado, 2019). Asimismo, la falta de sensibilización de los sistemas políticos respecto del tema ha llevado a esta problemática a ser considerada como un tema tabú, en lugar de ser considerada como un foco que debe ser atendido. Actualmente solo 38 países en el mundo han notificado contar con estrategias nacionales en la prevención del suicidio (OMS, 2019).

De igual manera, la falta de información y estudio respecto de la prevalencia del suicidio, suele ser una problemática que no permite el desarrollo efectivo de estrategias de prevención. En el caso de México, no se tiene una institución oficial que 
se encargue de agrupar estadísticas a nivel nacional respecto de los intentos de suicidio (Córdova, Rosales y Rosales, 2018).

Un aspecto a destacar, es que las variaciones en los patrones de suicidio cambian de país en país. Es decir, las características, causas y métodos de suicidio son distintos en cada país, lo que imposibilita la unificación de estrategias de prevención universales (OMS, 2018). Esta particularidad en cuanto al suicidio no solamente varía de país en país, sino de sujeto en sujeto; cada uno tiene sus propios motivos para ello.

En concordancia con esta diferenciación cultural de los patrones de suicidio, será importante apuntar a la necesidad de ubicar el riesgo suicida en su aspecto psicosocial, en donde el sujeto no se muestra como un ente individual y asocial, excluido de su entorno y contexto, sino por el contrario, responde a un sujeto singular, en donde la singularidad apunta a la construcción de un ser único y diferenciado de otros, pero a la vez formado e incluso definido como singular en su diferenciación y similitud con los otros (Lacan, 1936/2009). De esta manera, también el trabajo clínico (sobre todo de perspectiva psicoanalítica) deberá apuntar a este ser singular, tomando en consideración en todo momento su historia, su palabra, y sus deseos.

\section{PREVENIR EL SUICIDIO}

En muchas ocasiones, los programas preventivos se centran en intervenciones semanales o mensuales entre equipos de trabajo, o sesiones escolares con padres de familia, etcétera. Sin embargo, existen otros medios como la identificación del riesgo suicida realizada por medio de redes sociales gracias a la configuración de algoritmos que permiten, mediante las publicaciones de los usuarios, identificar patrones lingüísticos que indiquen un posible riesgo suicida e, incluso, la posible realización de un suicidio, así como análisis clínicos-lingüísticos de notas suicidas (Jiménez-Féliz y Caballero, 2010; Fernández-Cabana, 2015). Actualmente, plataformas y sitios web, particularmente redes sociales como Facebook, Tumblr, Twitter e Instagram, hacen uso de algoritmos que les permiten identificar patrones lingüísticos que puedan resultar en una señal de posible intento de suicidio. Esto señala que la prevención del suicidio en tiempo real es un objetivo dirigido por el desarrollo de la tecnología y la innovación (Bentley, Kleiman, Elliott, Huffman y Nock, 2018; Campo-Arias y Suárez-Colorado, 2019).
Según Pérez (2012), las redes sociales fungen como "una prolongación de las relaciones personales en la vida real, donde se forman comunidades como la familia, el colegio, o los compañeros de trabajo" (p. 59), por lo que estas se han convertido en un medio o sistema en donde los jóvenes (aunque no solamente los jóvenes) logran expresarse. Sin embargo, a pesar del impacto que las redes sociales han tenido en la modificación de las interacciones sociales, son pocos los estudios que apuntan a profundizar en las características del suicidio manifiestas mediante las redes sociales (Urrego et al., 2016). Todos los esfuerzos dirigidos por el desarrollo de tecnologías para la prevención del suicidio, apuntan hacia una dirección: la escritura. En este sentido, la escritura exhibida por medios digitales es un tipo de discurso que no debe pasar inadvertido, pues anula el encuentro directo con el otro, y de manera privada (luego pública) deviene la intimidad de la escritura como expresión del afecto.

\section{LA ESCRITURA COMO UNA VÍA ARTÍSTICA DE EXPRESIÓN DEL DOLOR PSÍQUICO}

Himes (2001) plantea que la escritura es una posibilidad de hablar desde donde el sujeto queda atrapado, y donde la locura del escritor es revelada como un acto de violencia contra el mundo exterior. De igual manera, esclarece que la escritura no es posible sin la presencia de un lector, el que se ve seducido por el discurso escrito, generando una interdependencia entre lenguaje y sociedad, escritor y lector. Una seducción que, al ser puesta en palabra, produce una relación directa y tensa con el deseo del autor.

El estudio de la escritura ha sido abordado desde diversas perspectivas o áreas de estudio, como la lingüística, filosofía, antropología, sociología, entre otros. De esta se ha reflexionado acerca de las dimensiones que atraviesa, así como su origen, evolución, los diversos sistemas de escritura, su función en la sociedad, así como su transmisión mediante la enseñanza y su aprendizaje. Así como otras áreas de estudio han centrado su interés en la escritura, la psicología también ha denotado interés por esta. Algunos estudios (Pennebaker, 1997) han probado la efectividad terapéutica de la escritura y giran en torno a la función de lo escrito, particularmente las implicaciones respecto del sujeto a partir del acto de escribir, los cambios cognitivos 
existentes en el acto de escribir, así como indicadores psicológicos y conductuales implicados en la terapéutica de la escritura.

El origen del estudio del arte y la cultura desde la psicología, tiene principio con los escritos de Freud. A lo largo de la obra freudiana se esclarece la similitud entre los sueños, los síntomas, el juego y el proceso creativo; todos tienen en común la satisfacción parcial de deseos. Por intermedio del juego el niño establece su primera creación artística -crea y recrea-, de lo interno, de los objetos perdidos. En este sentido, el juego es una función reparadora ante el duelo que permite la creación de un espacio transicional entre la fantasía y la realidad, donde lo externo queda suspendido temporalmente (De Tavira, 1996). En el sueño también se recrea una historia, sin embargo, en el arte, esta recreación es una simulación y tiene como consecuencia la creación de un objeto físico; es decir, lo interno del sujeto se hace manifiesto en el mundo real, se vuelve tangible. Asimismo, la diferencia entre sueño y arte radica en la inversión de la energía, dirigiéndose hacia nuevas significaciones en lugar de hacerlo hacia objetos del pasado. De esta manera, la escritura podría fungir de espacio de redistribución libidinal, y, al mismo tiempo, de mostración del estado subjetivo, haciendo uso del juego simbólico que el lenguaje posee, las posibilidades metonímicas (de condensación de la palabra de múltiples elementos inconscientes), y metafóricas (de desplazamiento de la palabra hacia nuevos significantes). Escribir, en esta propuesta psicoanalítica, no es solo proveer un espacio de explayamiento de la subjetividad, sino una posibilidad re-ordenadora de la realidad, que la palabra provee, que la propia producción ordenada y sublimada proporciona.

Las obras de arte fungen como medio de comunicación por medio de la simbólica del lenguaje. $\mathrm{El}$ arte favorece el reconocimiento de sí mismo en el otro, lo que se denomina conciencia humana. La simbolización es una actividad creadora que surge cuando el consciente alcanza un alto nivel de integración. La realidad es percibida por los sentidos y representa la realidad inteligible (De Tavira, 1996).

En el ámbito artístico, por ejemplo, llaman la atención los escritos de aquellos que se desempeñan en este ámbito y que llevan a cabo el suicidio. En su mayoría, estos manifiestan la inconformidad con la sociedad en la que se desenvuelven, conflictos sociales y privados, desesperanza, estados de ánimo melancólicos, entre otros. Sin embargo, este aspecto que resalta el involucramiento de los procesos psíquicos no es algo contemporáneo.

$\mathrm{El}$ arte se ha considerado desde tiempos remotos como una forma de expresión que procede del inconsciente. Una forma creativa y muy particular de hablar y de ser escuchado. Es decir, el proceso creativo (arte) puede considerarse como un significante de los actos para generar nuevos objetos, como la capacidad de encontrar soluciones nuevas u originales y como la voluntad de modificar o transformar el mundo: una especie de sostén o salvación. Asimismo, la creatividad puede referirse a un proceso o característica de la personalidad o de determinados productos humanos, donde los componentes emocional y relacional son base para su establecimiento y desarrollo (Jackson y Magagna, 2017). La escritura referida al suicidio, o anunciadora del acto suicida, ha sido parte fundamental de la creación, subjetividad y reconocimiento de la posición del sujeto creador en muchos/as grandes artistas, por poner algunos ejemplos: Virginia Wolf, Alejandra Pizarnik, Ernest Hemingway, Silvia Platt; o anuncios de la proximidad de la muerte mediante epistolarios literarios como Frida Kahlo, Tolstoi, entre otros. Algo que debe destacarse, es que no se requiere ser un artista reconocido para poder crear algo a partir del dolor psíquico y para que aquello que se ha creado posea las características que permitan desempeñar las funciones de este nuevo objeto.

Freud (2013) refiere la escritura como una tramitación sublimada de la pulsión, y describe al creador literario como un transformador de aquello que puede resultar penoso o innombrable para el mundo; lo determina como aquel que produce creaciones para generar un lazo con el espectador: "muchas cosas que de ser reales no depararían goce pueden, empero, depararlo en el juego de la fantasía" (Freud, 2013, p. 128). Ante esto, el texto adquiere el valor de un medio en el que se tramita la pulsión por medio de la sublimación y que, además, puede posibilitar el intento de la creación de lazos o como una vía de expresión de aquello que aqueja al sujeto. En este sentido, la escritura no funge como mera comunicación, sino como reordenamiento subjetivo, y de ahí que radique la importancia de pensarla como posible herramienta clínica en la prevención. Escribir es producir, escribir supone una construcción, tal y como lo expone Freud en relación con el ordenamiento delirante en el creador literario (Freud, 1907/2006). 


\section{LA CREATIVIDAD Y SUS IMPLICACIONES}

La Gradiva de Jensen, expuesta por Freud (1907/2006), establece las bases para pensar la escritura como herramienta de ordenamiento subjetivo. El autor de la obra explaya de manera delirante su pasaje a la locura, su encuentro con lo innombrable de la sexualidad y de la muerte misma. Haciendo uso del lenguaje creativo, de la creación de la obra literaria, Jensen expone lo que Allouch denominará "el paso a otra cosa", el abandono de la repetición mortífera de lo que retorna incansablemente en los pensamientos de muerte (Freud, 1920/2006), que la escritura anuda, como también lo mostrará Lacan en el seminario "El Sinthome", sobre James Joyce y la aparición del lenguaje en tanto lo simbólico que lo salva de la muerte (Lacan, 1975). Haciendo alusión a los clásicos registros del sujeto expuestos por Lacan (1953), lo simbólico emerge para cambiar el estatuto de lo Real hacia una forma ante la cual el sujeto pueda hacer algo, haciendo uso de su creatividad, como creación misma de algo diferente a la única posibilidad que se anuncia, la muerte. Más allá de la interpretación discursiva en un dispositivo clínico de escritura del suicidio, entraríamos al campo de la producción literaria que permite un ordenamiento distinto en la realidad del sujeto, por medio de la creatividad y del llamamiento al juego simbólico.

La creatividad es formadora ante cualquier expresión de arte y, suele entenderse como algo único y propio del ser humano. Los griegos consideraban la creatividad como algo sobrenatural, como vínculo con lo divino inspirado por las musas. En la Antigüedad, los poetas eran los intérpretes de los dioses, una vía que utilizaban los dioses para comunicarse con los mortales. Desde la psicología, particularmente desde el psicoanálisis, el asunto no es tan distinto (De Tavira, 1996).

El proceso creativo es entendido como la creación de cualquier forma de arte o expresión artística: pintura, escritura, escultura, danza, teatro, etcétera. Desde algunas posturas, la creatividad es concebida como el proceso de incubación que se produce del trabajo mental inconsciente; que es plasmado de forma manifiesta mediante una nueva creación. Freud conceptualiza la relación presente entre la creación artística y el inconsciente: una manifestación del proceso primario. Por su parte, Winnicott enfatiza el factor cultural como fuertemente influyente, asimismo, la experiencia artística es concebida por el autor como objeto transicional, como modo infantil para la resolución de conflictos (psíquicos). Para Klein, por otro lado, el arte es un medio de reparación (De Tavira, 1996). Todos coinciden en que el objeto creado (un texto, por ejemplo) posee una función relacionada al alivio del dolor psíquico, o a la tramitación de aquello que aqueja al sujeto.

Melanie Klein (1929) propone conceptos que clarifican los estadios que atraviesan los niños hasta alcanzar la adultez, concepto fundamental para el origen de la creatividad. Klein (1929) estipula que los impulsos agresivos pregenitales se expresan por medio de las fantasías inconscientes dirigidas hacia la madre. Este deseo sádico del cuerpo de la madre genera angustia, surgiendo el deseo del niño por reparar el daño de la madre; es precisamente este deseo de reparar lo que subyace al proceso creativo.

Un factor clave en el proceso creativo es que el artista posee capacidad para seleccionar derivados de sus conflictos psíquicos (propios y de otros). Freud (2013) señala que el Yo tiene como tarea remover lo necesario de la idiosincrasia y condicionamientos del artista para la inspiración. El artista debe ser capaz de tolerar lo que en sí mismo son fantasías universales, y debe ser capaz, en su proceso de elaboración secundaria de esas fantasías, para encontrar en ellas formas de expresión personal-original, una expresión que sea un soborno para el placer y permita ofrecer orden y tolerancia al Yo, para la exploración de identidades y experiencias de otra forma.

Para Klein (1929), la fantasía tiene un sentido motivacional: implica un deseo. El proceso creativo parte de las alucinaciones al servicio de las necesidades, y estas dan lugar a fantasías que son el origen de dicho proceso; por ejemplo, el fantaseo del suicida. Asimismo, Winnicott (1979) considera precisa la percepción creativa de la realidad, de forma que la vida posea un sentido. Para Winnicott, es necesario entender cómo la creatividad funciona como fenómeno transicional que permite al individuo hacer frente a la pérdida de la omnipotencia, siendo mediante un nuevo símbolo que el creador representa la separación madre-hijo, fungiendo el objeto artístico como una forma de unión. Winnicott expone que en el proceso creativo se anula la escisión entre sujeto y objeto; le posibilita al sujeto la oportunidad de encontrar un lugar u objeto que le permita sostenerse ante lo que le aqueja. 
El arte supone un medio de resolución, de tramitación ante lo que no puede ser tramitado de ninguna otra forma. De lo que aparece en el exceso, de lo imposible de tramitar. Se presenta algo inconsciente que empuja por expresarse. Un impulso innato necesario, que estimula la producción y creación para resolver lo que aqueja al sujeto. En cada producción escrita se escabulle una porción de inconsciente, que irrumpe de forma inesperada. El artista necesita escribir, pintar... necesita de un espacio mental por donde pueda correr en círculos para intentar dar sentido al dolor psíquico. Quien se expresa mediante el discurso artístico, expone en su obra o creación un lenguaje que es y ha sido imposible de hablar, que nunca ha sido compartido.

Ante esto, el arte adquiere un estatuto de actividad sublimatoria ante la tramitación de lo que no puede ser enunciado, modificando al sujeto desde lo más íntimo. Es menester mencionar en este punto que la escritura se ciñe a este proceso sublimatorio con la direccionalidad de aquello que es señalado por la angustia. Es precisamente por medio del texto que el escritor da cuenta de su angustia escribiendo de manera indirecta sobre el papel; es decir, que el lenguaje opera como un refugio en la tramitación de la angustia. Precisamente, la angustia precede al acto, ya que toda actividad humana engendra certeza, y es donde el sujeto pasa a ser representado por el significante. "Es quizás de la angustia de donde la acción toma prestada su certeza" (Lacan, 2013, p. 88). A partir de este punto, es preciso estipular el valor del acto de la escritura y su relación con la subjetividad de quien se expresa, así como destacar la importancia que la escritura conlleva en la prevención del suicidio.

\section{LENGUAJE Y PREVENCIÓN DEL SUICIDIO}

Diversos autores han intentado desarrollar bases de datos, fórmulas matemáticas, sistemas operativos, entre otras herramientas, que intenten identificar a los sujetos que presenten conducta suicida. Sin embargo, la particularidad del uso de estas herramientas se limita al ámbito forense o bien al uso de las tecnologías de comunicación. En la actualidad existen diversas herramientas cuantitativas y cualitativas para el análisis de textos con la finalidad de identificar si existen patrones lingüísticos entre las personas que llevan a cabo el suicidio.
Fernández-Cabana, García-Caballero, AlvesPérez, García-García y Mateos (2013) analizaron el libro Fragmentos, el que incluye notas, cartas y poemas escritos por Marilyn Monroe. En su estudio encontraron que los textos mostraban un uso consistente de altos porcentajes de pronombres singulares en primera persona (yo) y poco uso de pronombres plurales en primera persona (nosotros), lo que de acuerdo con estudios previos que vinculan ese patrón lingüístico, se relaciona con una sensación de aislamiento y un bajo nivel de integración social. De acuerdo con los autores, esto podría estar relacionado con la identidad del suicida.

Además, Fernández-Cabana et al. (2015) encontraron que quienes tienden a comunicar mediante notas su intención de suicidarse, suelen ser jóvenes y suelen no presentar diagnósticos previos de alguna alteración mental. En cuanto al análisis lingüístico, los autores señalan diferencias en el discurso de acuerdo con el género y el contexto de origen de los autores.

Es importante señalar que los estudios anteriormente mencionados hacen uso de herramientas cuantitativas que persiguen el objetivo de intentar identificar patrones lingüísticos entre suicidas, sin embargo, estas herramientas se centran en el conteo de uso de palabras; en su frecuencia. En este sentido, el lenguaje figurado, es decir, la particularidad del discurso escrito, no puede ser analizado mediante el uso de este tipo de herramientas (Barroso, 2017). Por tanto, esto excluye a su aplicación para el análisis de textos que incluyan el uso de la metáfora como base principal de expresión, por ejemplo, la poesía. La metáfora es entendida como un recurso retórico que puede ser comprendido como una palabra que reemplaza o que se asemeja a un aspecto de la realidad. Es decir, es una representación de un referente externo (Ricouer, 2010).

Si bien la escritura es un acto que busca el sostén de la vida de quien se expresa, el acto de escribir puede ser considerado también como un síntoma, por su característica de ser una manifestación del conflicto psíquico. La concepción del síntoma entrama una posición subjetiva y particular de sufrir que vela en lo más íntimo del sujeto y su verdad. Sin embargo, ante esta verdad, debido a que se trata de una subjetiva, es preciso intentar dilucidar de qué se trata, cuál es su importancia en un proceso de cura e identificar qué es aquello singular a lo que apunta. 
El síntoma es para Freud, al igual que el sueño, portador de un sentido susceptible de ser descifrado - un texto para ser leído-, lo que permite acceder al inconsciente del sujeto; además, vehiculiza la paradójica satisfacción al ser vivenciada por el sujeto como displacer. El síntoma si bien se articula con un querer decir, también se articula con lo indecible. Este indecible entra en relación con la satisfacción pulsional que tiene lugar desde el Ello. Freud utiliza la metáfora al comparar el síntoma con una perla, la que se forma a partir de un grano de arena, y donde las capas de la perla son el equivalente al endosamiento de los diversos sentidos del inconsciente (Dávila, 2016).

Es precisamente esto indecible aquello que debe ser descifrado, aquello que podría dar pautas para un indicio de conducta suicida o bien para prevenir la consumación de un acto suicida. La poesía "es un lenguaje cuya palabra ha de ser liberada" (Anzieu, 1981) y que se presenta de manera impulsiva, tal como ocurre con los síntomas neuróticos. De igual manera, Cadoux (1999) hipotetiza que la escritura viene a suplir un fallo en el aparato psíquico, dando lugar a lo que el sujeto no puede elaborar en sí mismo. Esto supone una inscripción externa de algo que no puede inscribirse en el sujeto; un trabajo que persigue la contención y la elaboración del yo. De aquello que intenta nombrarse y que, a pesar de sus esfuerzos, no encuentra salida más que por medio de la palabra escrita.

Los medios actuales de interpretación de textos que proponen la identificación de la conducta suicida pueden resultar ineficientes, ya que se limitan a algoritmos o metodologías cuantitativas, excluyendo cualquier tipo de lenguaje metafórico. De igual manera, es preciso mencionar que el lenguaje pone en evidencia la verdad de aquello que aqueja al sujeto y es por ello que debe ser considerado como una herramienta de la que debe hacerse uso para la prevención del suicidio.

La creación artística es un espacio donde el artista es capaz de plasmar aquello que pertenece al orden de lo desconocido por la conciencia. La escritura, como manifestación de la creación artística, evidencia la expresión del inconsciente mediante la palabra textual: la expulsión autónoma del sujeto plasmada en un cuerpo de papel (Himes, 2001). $\mathrm{Y}$ es precisamente por esta característica que se puede tomar ventaja de ello para la identificación de aquellos sujetos que puedan manifestar el deseo de terminar con su vida.
La escritura es una vía de acceso hacia el conocimiento de aquello que aqueja al sujeto (Artières, 2016). Si bien es cierto que el suicidio no puede ser prevenido de manera sencilla, es posible identificar algunas características en el discurso de aquel que se encuentra en riesgo suicida. Es por ello que los esfuerzos deben dirigirse a la identificación de la particularidad del discurso del sujeto suicida y no limitarse al conteo de palabras que apunten hacia su relación con el suicidio en los análisis forenses o a simples anuncios de advertencia cuestionando al usuario de alguna red social si presenta algún problema.

A pesar de que es posible pensar en sujetos que por la escritura logran sostenerse exitosamente, formulando soluciones que les permiten sostenerse para enfrentar aquello que los aqueja, no en todos los casos esto suele suceder. Basta con considerar las notas y diarios de los suicidas para dar cuenta de que la expresión escrita del padecer, en muchas ocasiones no suele ser suficiente para sostener al sujeto que sufre. Ante esta problemática, es menester de que todo aquel que pueda acceder a los textos de pacientes en riesgo, sea capaz de identificar lo que el sujeto expresa realmente; ir más allá de las posturas tradicionales respecto del arte, en este caso de la escritura.

\section{CONSIDERACIONES FINALES Y PERSPECTIVAS A FUTURO}

El presente trabajo, en su carácter exploratorio y teórico con base psicoanalítica, busca exponer a la escritura como una posibilidad real de herramienta ante el discurso del sujeto suicida. Si bien es cierto que existe literatura que apoya con datos la efectividad terapéutica de la escritura, aquí mostramos una posibilidad no solo terapéutica, sino de auténtico reposicionamiento subjetivo de quien se muestra a sí mismo como poseyendo ideas suicidas. El presente trabajo establece las bases para un diseño posterior de intervenciones clínicas a nivel escritural, o bien, de la atención a las producciones creativas del sujeto suicida. Es necesario considerar desde dónde escribe el sujeto y la función que esto conlleva respecto de este, de manera que puedan realizarse intervenciones puntuales y pertinentes con la finalidad de perseguir la prevención del suicidio en distintos ámbitos, y no solamente en el sector salud, porque la prevención del suicidio debe ser desestigmatizada y considerarse como una problemática social real, que tiene repercusiones 
no solamente en las familias de aquellos que consuman el suicidio, sino en todos los ámbitos que involucran a una nación.

La escritura es una vía por donde los procesos psíquicos de las personas se hacen manifiestos. Es por ello que debe destacarse su importancia tanto en el ámbito de la prevención como de las intervenciones que apuntalan hacia la problemática del suicidio. De igual manera, es importante mencionar la poderosa herramienta que puede resultar el discurso escrito en el ámbito clínico, no solamente como parte de la terapia de los pacientes, sino además como una forma más de generar el acercamiento con los procesos psíquicos del paciente; por ejemplo, la creación autónoma de diarios, poemas, entre otros escritos por parte de los pacientes, pueden derivar en un acercamiento con las manifestaciones de riesgo suicida y la conducta suicida: un intento de hablar de aquello que no puede expresarse con facilidad por diversos motivos. Desde esta perspectiva, es importante destacar la importancia de las herramientas escriturales y los diferentes tipos de análisis de discursos que existen y que pueden ser de gran utilidad en el ejercicio de la prevención (por mencionar algunos: análisis interpretativo, análisis fenomenológico, análisis lacaniano de discurso, entre otros). Un análisis de este orden, de la producción escrita del sujeto suicida, permitirá una terapéutica, pero también un posible reordenamiento subjetivo por medio del uso de la creatividad y la convocatoria a la sublimación, o a un fragmento de ella.

La incorporación de la escritura como una herramienta a la identificación de personas en riesgo de suicidio, puede ser efectiva. Desgraciadamente, el incremento de los suicidios, así como de la tendencia a la generalización de un discurso denominado "suicida", pueden limitar la eficacia de la interpretación de los textos. El discurso es único en cada sujeto y, como tal, debe ser interpretado o analizado de la misma manera. Si bien algunas plataformas virtuales han sido eficaces para detectar intentos de suicidio, no lo han sido para prevenirlos realmente; sino simplemente para identificar a quien ya ha hecho manifiesta su intención explícita de suicidarse o de estar sufriendo.

La escritura y la interpretación de esta es una vía de comunicación, de enunciación del estado subjetivo, y una forma de crear un vínculo entre la sociedad en general con el que sufre. Es una herramienta que sirve para ser leído el síntoma (en el sentido psicoanalítico del término, en donde el síntoma es una articulación emergente e inconsciente del lenguaje, que rebasa los límites de la palabra). El síntoma ansía ser leído y, en muchas ocasiones, también el sujeto que sufre y que no encuentra posibilidad alguna ante su propio dolor.

Si bien el presente trabajo pretende abordar algunas consideraciones acerca del uso de la escritura como herramienta clínica para la prevención del suicidio, dentro del campo del psicoanálisis sería de utilidad indagar en futuros estudios la producción artística-literaria de autores/as que han consumado el suicidio, lo que permitiría explorar el alcance o las funciones de la creación artística como expresión metafórica o real de la posición subjetiva. Del mismo modo, el presente trabajo podría prestarse a manera de precedente para una indagación futura acerca de las múltiples formas de análisis de discursos escritos concernientes al suicidio, y sus alcances dentro y fuera del contexto de la clínica, tanto para la identificación y prevención del riesgo suicida, destacando en todo momento la singularidad del caso. Si bien el presente trabajo se enfoca en una perspectiva desde el psicoanálisis, un estudio más completo acerca de la cuestión podría darse privilegiando metodologías cualitativas y discursivas diversas, donde la interpretación o la construcción de casos pueda apuntar a diversos alcances y contextos, más allá de los casos clínicos que pudieran presentarse a los profesionales de la salud. 


\section{REFERENCIAS}

Artières, P. (2016). Clínica de la escritura: historia de la mirada médica sobre la escritura. Barcelona: Editorial Gedisa.

Anzieu, D. (1981). Psicoanálisis y Lenguaje, del cuerpo a la palabra. Buenos Aires: Editorial Kapelusz.

Barroso, A. A. (2017). Alcances y limitaciones de un enfoque de riesgo para comprender el suicidio. Revista Hospital Psiquiátrico de la Habana, 14 (1), 12-24, https://www.medigraphic.com/ cgi-bin/new/resumen.cgi?IDARTICULO=78398

Bentley, K., Kleiman, E., Elliott, G., Huffman, J. y Nock, M. (2018). Real-time monitoring technology in single-case experimental design research: opportunities and challenges. Behavior Research and Therapy, 117, 87-96, https://www.sciencedirect. com/science/article/pii/S0005796718301980?via\%3Dihub

Cadoux, B. (1999). Écritures de la psychose. Paris: Aubier.

Campo-Arias, A. y Suárez-Colorado, Y. (2019). ¿Es el suicidio un evento prevenible? Rev. Univ Ind Santander Salud, 51, 197-199, https://revistas.uis.edu.co/index.php/revistasaluduis/ article/view/9663

Cervantes, W. y Melo, E. (2008). El suicidio en los adolescentes: Un problema en crecimiento. Duazary, 2, 148-154, http://revistas.unimagdalena.edu.co/index.php/duazary/article/view/669

Córdova, M., Rosales, J. C. y Rosales, A. (2018). Intento suicida: Veinticinco años de investigación en estudiantes mexicanos de educación media superior. Revista Digital Internacional de Psicología y Ciencia Social, 5, 176-198, http://cuved.unam. $\mathrm{mx} / \mathrm{rdipycs} / \mathrm{p}=6990$

Dávila, K. (2016). La orientación por el síntoma. Desde el jardín de Freud, 16, 53-62, https://revistas.unal.edu.co/index. php/jardin/article/view/58152

De Tavira, F. (1996). Introducción al Psicoanálisis del arte. México: Plaza y Valdés.

Fisher, A.J., Medaglia, J.D., Jeronimus, B.F. (2018). Lack of group-to-individual generalizability is a threat to human subjects research. Proceedings of the National Academy of Sciences, 115, 1-10, https://www.pnas.org/content/115/27/E6106.long

Fernández-Cabana, M. (2015). Suicidio y escritura: Un análisis clínico-lingüístico de textos y notas suicidas (Tesis de doctorado). Universidad de Santiago de Compostela, Santiago de Compostela, España.

Fernández-Cabana, M., Jiménez-Féliz, J., Alves-Pérez, M. T., Mateos, R., Gómez-Reino Rodríguez, I. ... García Caballero, A. (2015). Linguistic analysis of suicide notes in Spain. The European Journal of Psychiatry, 29, 67-77, http://scielo.isciii. es/pdf/ejpen/v29n2/original6.pdf

Fernández-Cabana, M., García-Caballero, A., Alves-Pérez, M. T., García-García, M.J. y Mateos, R. (2013), Suicidal Traits in Marilyn Monroe's Fragments. Crisis, 34, 124-130 doi: 10.1027/0227-5910/a000183

Freud, S. (2013). El creador literario y el fantaseo. En J. Strachey (Ed.), En Obras Completas. Tomo IX. Buenos Aires: Amorrortu.

Freud, S (1907/2006). El delirio y los sueños en la Gradiva de W. Jensen, En Obras Completas IX. Buenos Aires: Amorrortu.

Freud, S (1920/2006). Más allá del principio de placer, En Obras Completas XX. Buenos Aires: Amorrortu.
Himes, M. (2001). Beyond the pleasure of the text: the writer and the reader. Psychoanalysis y Contemporary Thought, 24 (3), 15-22

Jackson, M. y Magagna, J. (2018). Creatividad y estados psicóticos en personas excepcionales. Barcelona: Herder Editorial.

Jiménez-Féliz, J., Caballero, G (2010). Características forenses, psicológicas y lingüísticas de una muestra de notas suicidas en Galicia. Boletín gallego de medicina legal y forense, 17, pp. 31-47.

Klein, M. (1929). Situaciones infantiles de angustia reflejadas en una obra de arte y en el impulso creador. Obras Completas, III. Buenos Aires: Paidós.

Klonsky, E., May, A. y Saffer, B. (2016). Suicide, suicide attempts, and suicidal ideation. Annual Review of Clinical Psychology, 12, 307-330, https://www.annualreviews.org/doi/full/10.1146/ annurev-clinpsy-021815-093204?url_ver=Z39.88-2003\&rfr_id=ori\%3Arid\%3Acrossref.org\&rfr_dat=cr_pub\%3Dpubmed

Klonsky, E., Saffer, B., Bryan, C. (2018). Ideation-to-action theories of suicide: a conceptual and empirical update. Current Opinion in Psychology, 22, 38-43, https://www.sciencedirect. com/science/article/pii/S2352250X17301835?via\%3Dihub

Lacan, J (1936/2009). El estadio del espejo como formador de la función del yo (je), tal como se nos revela en la experiencia psicoanalítica, En Escritos I. México: Siglo XXI.

Lacan, J (1953). Lo simbólico, lo imaginario y lo real. Conferencia dictada en el Hospital de St. Anne, https://www.lacanterafreudiana.com.ar/2.5.1.4\%20\%20\%20LO\%20SIMB,\%20LO\%20 IMAG\%20Y\%20LO\%20REAL,\%201953.pdf

Lacan, J (1975). Seminario 23. El Sinthome. Buenos Aires: Paidós.

Lacan, J. (2013). Seminario 10. La angustia. Buenos Aires: Paidós.

Mann, J., Apter, A., Bertolote, J., Beautrais, A., Currier, D. ... Haas, A., (2005). Suicide prevention strategies: a systematic review. JAMA, 294, 2064-2074, https://jamanetwork.com/ journals/jama/article-abstract/201761

Ministerio de Salud del Gobierno de Chile (2013). Programa nacional de prevención de suicidio. Orientaciones para su implementación. Recuperado de: https://www.minsal.cl/sites/ default/files/Programa_Nacional_Prevencion.pdf

Organización Mundial de la Salud (2001). Prevención del suicidio. Un instrumento para docentes y demás personal institucional. Recuperado de: https://www.who.int/mental_health/ media/en/63.pdf

Organización Mundial de la Salud (2014). Prevención del suicidio. Un imperativo global. Recuperado de: https://www. who.int/mental_health/suicide-prevention/exe_summary_spanish.pdf?ua=1

Organización Mundial de la Salud (2018). Suicidio. Recuperado de: who.int/topics/suicide/es/

Organización Mundial de la Salud (2019). Suicidio. Recuperado de: https://www.who.int/es/news-room/fact-sheets/detail/suicide

Pennebaker, J (1997). Writing about emotional experiences as a therapeutic process, Psychological Science, 8 (162). DOI: 10.1111/j.1467-9280.1997.tb00403.x 
Pérez, J. (2012). Estructura del mercado audiovisual: Resultados. España: Grupo de Investigación EUMED.

Ricouer, P. (2010). Del texto a la acción, ensayos de hermenéutica II. México: Fondo de Cultura Económica.

Schwartz-Lifshitz, M., Zalsman, G., Giner, L. y Oquendo, M.A. (2012). Can we really prevent suicide? Current Psychiatry Reports, 14, 624-633, https://link.springer.com/ article/10.1007\%2Fs11920-012-0318-3

Urrego, Y., Quintero, A. y Manrique, J. (2016). Caracterización de adolescentes con conductas suicidas a través de redes sociales; Facebook, Foros y Open-blogs. En M. Molero, M., Pérez-Fuentes, M., Gázquez, J., Barragan, A., Martos, A. ...
Simón, M. (comps.). Avances de Investigación en Salud a lo largo del Ciclo Vital (pp. 78-86). España: ASUNIVEP.

Winnicott, D. (1979). Realidad y Juego. Buenos Aires, Argentina: Gedisa.

Zalsman, G., Hawton, K., Wasserman, D., Van Heeringen, K., Arensman, E. ... Sarchiapone M. (2016). Suicide prevention strategies revisited: 10-year systematic review. Lancet Psychiatry, 3, 646-659, https://www.clinicalkey. com/\#!/content/playContent/1-s2.0-S221503661630030X? returnurl=https:\% 2 F\% 2Flinkinghub.elsevier. com\%2Fretrieve\%2Fpii\%2FS221503661630030X\%3Fshowa11\%3Dtrue\&referrer=https:\%2F\%2Fwww.ncbi.nlm.nih.gov $\% 2 \mathrm{~F}$ 